\title{
End-of-life Care in Postgraduate Critical Care Nurse Curricula: An Evaluation of Current Content Informing Practice
}

\author{
KristenRanse $^{1}$ \\ LoriDelaney $^{2}$ \\ JamieRanse $^{1}$ \\ FionaCoyer ${ }^{2}$ \\ PatsyYates $^{2}$
}

Introduction: Despite the frequency with which critical care nurses' engage in end-of-life care, preparation and support of nurses for this work through professional development opportunities or postgraduate education is reportedly limited.

Objectives: To identify and describe end-of-life care content in postgraduate critical care nursing courses in Australia.

Methods: A purposive sampling technique was used to invite a postgraduate course convener from each higher education institution, identified through a search of the internet, offering a postgraduate course in critical care nursing $(n=17)$. An individual structured telephone survey was undertaken with each participant $(n=13)$. Descriptive statistics and content analysis of participant responses was undertaken to identify and describe the end-of-life content and modes of content delivery.

Results: Participants agreed (92\%) that end-of-life care content was important within post-graduate education, with $77 \%$ agreeing that more time should be allocated to focus on end-of-life care. The majority of courses addressed legal and ethical issues related to end-of-life care $(77 \%)$, however this content was predominately covered in the context of organ donation (92\%). The content least commonly addressed pertained to the work of the nurse in providing direct clinical care, including the physical changes experienced by the dying patient (31\%), the process of withdrawing life sustaining treatment (15\%), withdrawal of ventilation and symptom management (23\%), emotional support to the family (23\%) and care of the body after death (23\%).

Conclusion(s): The inclusion of end-of-life care content in critical care curricula is needed to address the complexity of this area of practice, including the care and inclusion of family, the physical care and emotional support of the patient and self-care for the nurse. It is recommended that 
targeted educational initiatives are implemented to improve the care that patients and their families receive and support nurses in the delivery of this care. 